\title{
Impacto de secuestros y homicidios en la inversión extranjera directa en México
}

\author{
Impact of kidnappings and homicides on foreign direct \\ investment in Mexico
}

\author{
Eduardo Loría* \\ Universidad Nacional Autónoma de México, México \\ Recibido el 18 de Octubre de 2018; aceptado el 9 de Mayo de 2019 \\ Disponible en Internet el: 21 de mayo de 2019
}

\section{Resumen}

Al menos desde 2006, México sufre un alarmante incremento de la criminalidad. Delitos de alto impacto como homicidios dolosos y secuestros han aumentado considerablemente. Desde el año 2000, la Inversión Extranjera Directa (IED) se ha estancado nominalmente y ha caído como proporción del PIB (de $3.9 \%$ en 2001 a $2.6 \%$ en 2017).

Con el uso de seis Modelos de Vectores de Corrección de Error se prueba empíricamente, con datos anuales para el periodo 1997-2017, el efecto negativo, permanente y significativo de la tasa de secuestros y de los homicidios dolosos sobre la IED Total, sus componentes y sobre el PIB. Se muestra que aplicar políticas eficientes que recuperen el Estado de Derecho es crucial para elevar la capacidad de crecimiento económico.

Código JEL: C32, C52, F21, K42

Palabras clave: Inversión extranjera directa y sus componentes; Secuestros; Homicidios dolosos; Vector de corrección de error (VECM)

\footnotetext{
* Autor para correspondencia Correo electrónico quijano6919@ hotmail.com (E. Loría).

La revisión por pares es responsabilidad de la Universidad Nacional Autónoma de México. 


\begin{abstract}
At least since 2006, Mexico suffers an alarming increase in crime. High impact crimes such as murders and kidnappings have considerably increased. Since 2000, the Foreign Direct Investment (FDI) has stagnated in current US dollars and fallen as a proportion of GDP (from 3.9\% in 2001 to $2.6 \%$ in 2017). By estimating six Vector Error Correction Models (with yearly data for the period 1997-2017), it is empirically proven the negative effect of the rate of kidnappings and murders on Total FDI, its components and the Mexican GDP. In all cases, the effects are permanent and significant. It is shown that policies focused on the recovery of the rule of law are crucial to raise economic growth.
\end{abstract}

Code JEL: C32, C52, F21, K42

Keywords: Foreign direct investment and its components; Kidnapping; Murder; Vector error correction (VECM)

Un Estado de Derecho efectivo reduce la corrupción, protege a las personas de injusticias, y combate la pobreza. El Estado de Derecho es el sustento de comunidades de igualdad, oportunidades, y paz, además que funge como la base del desarrollo, de gobiernos transparentes que rinden cuentas, y del respeto a los derechos fundamentales. [...] Cuando el Estado de Derecho es débil, no hay suficientes medicinas en las clínicas, la violencia y la delincuencia no se pueden controlar, la ley se aplica de forma injusta, y no hay inversiones extranjeras. El Estado de Derecho es un tema que no solamente involucra a abogados y jueces, sino que es un concepto que involucra a toda la sociedad.

World Justice Project, 2018: 10 


\section{Introducción}

La literatura convencional sobre la Inversión Extranjera Directa (IED) plantea que sus determinantes son esencialmente de tipo macroeconómico. Sin embargo, en años recientes y para algunos países emergentes se han añadido otras variables como la estabilidad política y social y la criminalidad, que apelan a aspectos microeconómicos y al entorno de las empresas.

La violencia que desde hace más de una década azota a México impacta a la paz social, pero también acarrea problemas económicos y la IED se ha visto afectada a partir del incremento de los delitos de alto impacto, medidos a través de las tasas de secuestros y de homicidios dolosos, que envían señales claras que desincentivan a los inversionistas y a los mercados internacionales, relegando los fundamentales macroeconómicos a un segundo plano.

El aumento de la criminalidad y la ausencia de estado de derecho elevan la inestabilidad social, deprimen la inversión privada y distraen (asignan a actividades no productivas) el gasto público y el privado y, en consecuencia, reducen la actividad económica. Todo ello genera un aumento de los costos económicos que consisten en la suma del valor total de los bienes y servicios usados para prevenir a los ciudadanos y aquellos que se asignan para tratar de inmediato a las víctimas. También hay otros costos asociados - más difíciles de medir - que se refieren a las múltiples consecuencias de largo plazo que acarrea como la elevación de las tasas de morbilidad (física, emocional y psicológica) y mortalidad (UNDOC, 2007) que, al tratarse de problemas de carácter público, generan externalidades que tarde o temprano el Estado debe tomar en sus manos con el consecuente costo presupuestario. ${ }^{1}$ Todo ello puede condenar a una economía a una trampa de bajo crecimiento.

El objetivo del trabajo es calcular el impacto que el incremento de la tasa de secuestros y homicidios dolosos tiene en el flujo de IED en México, en el periodo 1997-2017.

Se estimaron seis VECM(1) y, a partir de la precisión del pronóstico dentro de la muestra (evaluado con el Coeficiente de Theil) y por la correcta identificación (conjunta) y especificación, se eligieron cuatro para así detectar sus mejores determinantes y analizar la sensibilidad de largo plazo que tienen la IED, sus componentes y el PIB a esos delitos.

El análisis se hizo con series anuales debido a que sólo así las variables muestran homogeneidad en el orden de integración.

\footnotetext{
${ }^{1}$ Becker (1968: 171) y Rizzo (1979: 177 y 178) explican que, de acuerdo con la President's Comission on Law Enforcement and Administration of Justice, el costo del crimen consiste en la suma de el valor de mercado estimado de los bienes robados o destruidos, la pérdida de ganancias relacionada con muertes o lesiones y el gasto realizado por agentes privados y por el Estado para prevenir el crimen; por ejemplo, el gasto erogado en rubros como los distintos cuerpos de policía, juzgados, prisiones, abogados, alarmas anti robo y guardias, entre otros medios de protección. Además, Becker (1968: 171) considera que estos costos están altamente subestimados porque no toman en cuenta otros gastos que se hacen para prevenir un crimen; por ejemplo, el gasto en taxis para evitar asaltos en la vía pública y en el transporte público.
} 
Los resultados de la investigación son consistentes con la literatura reciente en el sentido de que la estabilidad social, la institucionalidad y la seguridad cada vez inciden de manera más importante en la entrada de IED a México y en el crecimiento económico.

Se demuestra que, aunque las variables macroeconómicas de la economía mexicana han sido estables, el incremento de la tasa de secuestros y de homicidios dolosos ha afectado negativa y permanentemente al flujo de la IED, sus componentes y al PIB en el periodo de estudio. En específico, se prueba empíricamente que su componente "Nuevas Inversiones" (IEDNI) - que refiere al "capital fresco" - ha venido cayendo de manera importante y la IED Total (IEDT) - que se ha mantenido estable en términos nominales desde 2000 - se explica centralmente por el rubro de "Reinversiones" (IEDR). Esto refleja que, por las condiciones de inseguridad, la entrada de flujos nuevos ha disminuido, aunque la IED ya establecida se retroalimenta porque parar sus actividades y salir del país resulta más costoso que quedarse y absorber el costo de la violencia. Además, como se explica en la sección de "Análisis de resultados", esta variable parece ser muy sensible a la evolución del mercado interno, por lo que el crecimiento económico reciente, aunque modesto, ha servido para contrarrestar el efecto negativo de la violencia en la IED.

También se encontró que la IEDR, que es el componente que ha crecido de manera relativa, tiene efectos positivos pero transitorios sobre el PIB, probablemente porque tiene escasos efectos de acumulación de capital.

Asimismo, llama la atención - y es lo más preocupante - la capacidad del crimen de auto reproducirse. En todos los casos, Sec y Hom muestran histéresis, lo que da cuenta de que, al ser actividades rentables, quienes participan en ellas no tienen incentivos para salirse y dedicarse a otra actividad legal.

En conclusión, restablecer el estado de derecho es crucial para elevar la IEDT y su componente de nuevas inversiones y así reactivar el crecimiento económico del país.

El trabajo está dividido en seis partes además de esta introducción. La primera muestra brevemente la evolución de la IED en México desde 1970. En la segunda se hace una revisión de la literatura y se recuperan aspectos teóricos. La tercera presenta los hechos estilizados de las variables de interés en México desde 1997, que es a partir de ese momento que se tienen series oficiales sobre esos delitos. La cuarta presenta los aspectos econométricos. La sección siguiente analiza y discute los resultados estadísticos obtenidos. Por último, se presentan las conclusiones.

\section{Evolución de la IED en México}

A partir del inicio de la globalización, en la década de los 80, el flujo de IED hacia los países en desarrollo se incrementó notablemente y se convirtió en un complemento importante de 
la inversión nacional e incluso en el motor de crecimiento de varios países como Irlanda y China (Ríos-Morales y O’Donovan, 2006).

Las causas de este fenómeno han sido objeto de diversos estudios y han dado origen a diferentes teorías que tratan de explicarlas. Algunas mencionan la búsqueda de mejores condiciones de mercado, diversificación de riesgo, ampliación de mercados, tipo de cambio favorable, pérdida de competitividad en el país de origen, relocalización productiva y estratégica y mejor competencia entre grandes empresas por ampliar sus mercados y mantener condiciones de mercado que favorezcan sus intereses (Guerra, 2001 y Esquivel y Larraín, 2001).

Entre los beneficios que se le han atribuido a la IED están: a) la generación de empleos; b) la incorporación de nuevas tecnologías y la aplicación de mejores ideas y prácticas de trabajo; c) el fomento y la construcción de industrias que propician la competencia; d) el impulso a la investigación y el desarrollo; y e) ser fuente de financiamiento del déficit estructural de la cuenta corriente de la balanza de pagos.

En síntesis, la literatura convencional considera que la IED genera importantes efectos positivos de arrastre (spillovers) (Aitken et al, 1994; Cevis y Camurdan, 2009; Findlay, 1978; Kotrajaras, 2010; Waldkirch, 2010 y Dussel et al., 2003) y, por lo tanto, es un factor crucial del crecimiento económico.

Según la UNCTAD (2017), durante la década de los 90 el crecimiento de la IEDT en el mundo fue exponencial y solo en dos periodos muy específicos cayó dramáticamente. El primero fue entre 2000 y 2003, en que se redujo en 59\%. Según esa organización, esto se debió a recesiones en más de una docena de países, ${ }^{2}$ incluidas las tres economías más grandes del mundo. Posteriormente, la Gran Recesión nuevamente volvió a deprimirla y hasta 2016 no había alcanzado el nivel que tuvo en 2007.

En México, la participación de la IED en el PIB ha sido muy errática y ha tendido a caer desde hace casi veinte años. En efecto, hacia 2001 - que es el punto más alto de toda la serie- representó 3.9\% del PIB y en 2017 cayó hasta 2.6\%, cifra similar a la que tuvo en 1994, ver figura 1. Esta evolución sugiere que a pesar de todas las reformas estructurales aplicadas y aun cuando los fundamentales macroeconómicos han estado en orden, esta importante variable no ha ganado peso y por ello podría contribuir a explicar que no haya repuntado el crecimiento de la economía mexicana.

\footnotetext{
${ }^{2}$ Ocasionadas por la crisis punto com y por las consecuencias de los eventos del 11 de septiembre de 2001 en Estados Unidos.
} 


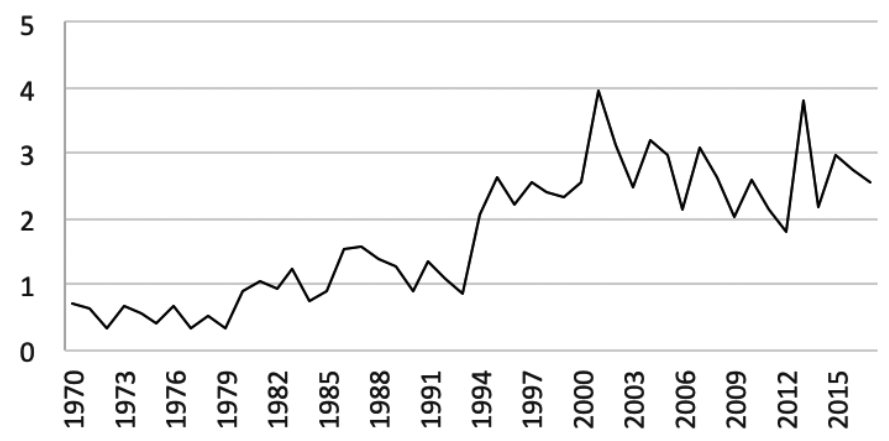

Figura1.México: IED como porcentaje del PIB, 1970-2017

Fuente: UNCTAD (2017).

Por último, la mayor parte de la IED en México se ha concentrado en las manufacturas y en los servicios financieros, lo cual no es fortuito debido a que el TLCAN justamente privilegió la liberación del comercio, la integración productiva regional y la liberación de las inversiones al vincular cadenas de valor de México y Estados Unidos en esos sectores, véase figura 2.

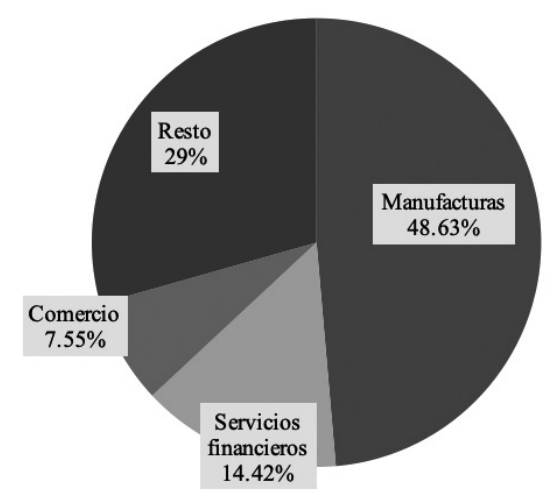

Figura 2.México: Distribución de la IED por sectores, 1999-2017

Fuente: Cálculos propios basados en Secretaría de Economía (2017). Promedio aritmético de la IED acumulada del periodo. Nota: Resto = Minería, Medios Masivos, Servicios Inmobiliarios, Servicios Turísticos, Construcción, Agricultura, Servicios Profesionales, Salud y Educación. 


\section{Revisión de literatura}

\section{Factores Macroeconómicos}

En la literatura convencional se encuentran coincidencias en cuanto a que los determinantes de la IED son esencialmente de orden macroeconómico como: el nivel de actividad y su tasa de crecimiento, el grado de apertura, el tipo de cambio, la inflación y alguna variable de riesgo país. En este sentido, Oladipo (2013) apunta al grado de apertura, el tipo de cambio, la oferta monetaria y la tasa de interés. Dussel et al. (2003) plantean que en México depende del crecimiento del PIB, del grado de apertura comercial, de los costos laborales del mercado receptor y del riesgo país medido como la proporción del déficit en cuenta corriente respecto del producto.

Fajardo y de Jesús (2015) mencionan que los factores que han determinado la IED en América Latina en años recientes son el PIB, la población económicamente activa, la inflación, la apertura comercial, la dinámica de crecimiento y el tamaño de mercado. Por su parte, Abbas y El Mosallamy (2016) encontraron que los determinantes de la IED para países emergentes son: el grado de apertura comercial, el capital humano, la infraestructura y el nivel de IED del periodo anterior. Por último, Dellis et al. (2017) sugieren que la calidad de las instituciones ${ }^{3}$ y la estructura económica ${ }^{4}$ son importantes para atraer IED en los países avanzados.

\section{Factores Microeconómicos}

Aspectos sociales como la criminalidad pueden ser incorporados como factores negativos en la determinación de la IED si consideramos que las empresas son aversas a incurrir en gastos de seguridad y, sobre todo, a ser víctimas de la delincuencia en cualquiera de sus formas.

Agyapong et al. (2016) confirman la existencia de efectos negativos del crimen organizado sobre el crecimiento en África. Mencionan que la inseguridad merma el flujo de IED debido a que el crimen actúa como un impuesto sobre toda la economía, reduce la competitividad al generar ineficiencias e incertidumbre que desincentivan la inversión doméstica y extranjera. Busse (2005) señala que en los países que presentan alta probabilidad de secuestro disminuyen las expectativas de retorno de los inversionistas.

Blomberg y Mody (2005) encontraron que en los países miembros de la Organización Mundial del Comercio (1980-2000), la violencia de todo tipo deprimió los flujos de comercio e IED. En ese sentido, Vittorio y Marani (2008) muestran que, debido a la presencia de grupos delictivos, el sur de Italia recibe una parte mínima de la IED que entra al país.

\footnotetext{
${ }^{3}$ Medida por el Índice Global de Competitividad y el Índice de Libertad Económica.

${ }^{4}$ Medida por los Costos Laborales, el Tamaño de Mercado y el Grado de Apertura.
} 
Pyshval y Suárez (2006) calculan que el incremento de $1 \%$ en la tasa de secuestros a personal de las firmas en Colombia trae consigo una disminución de la IED de $0.443 \%$. ${ }^{5}$ Ortiz et al. (2013) afirman que la tasa de secuestros, también en Colombia, ha tenido un efecto negativo y significativo sobre el crecimiento económico desde 1980.

\section{La criminalidad en México}

Para el caso específico de México hay trabajos recientes que incorporan variables de criminalidad en el estudio de la IED.

Madrazo (2009) relaciona la IED con crímenes, el PIB y el salario mínimo, y manifiesta que los homicidios dolosos la afectan significativa y negativamente. Alaimo et al. (2009) concluyen que el crimen tiene una incidencia importante y negativa en el desempeño de las empresas y afecta más a la inversión que a la productividad, por lo que sugieren que existe un nivel límite de violencia que las empresas están dispuestas a aceptar.

Bernal y Castillo (2012) afirman que los homicidios y secuestros tienen efectos negativos sobre la IED, sobre todo a partir del inicio de la "guerra contra el narcotráfico" en diciembre de 2006. ${ }^{6}$ Hernández (2014) concluye que esta estrategia de combate a la delincuencia (2006-2012) fue errada en el sentido de que exacerbó el círculo vicioso de la violencia. Torres y Polanco (2015) sugieren que el aumento de la tasa de homicidios reduce la capacidad de atracción de la IED.

\section{La IED y la violencia en México. hechos estilizados}

Como se mencionó, contrario a lo que buscaba esa estrategia, la criminalidad en todas sus manifestaciones ha crecido rápidamente. Según el Informe Regional de Desarrollo Humano de PNUD (2013) y Molzahn et al. (2012), a partir del inicio de la "guerra contra el narcotráfico" las tasas de homicidios y secuestros crecieron aceleradamente.

De acuerdo con ENVIPE (2017), durante 2016, el costo de la inseguridad fue de alrededor de $1.1 \%$ del PIB, donde el $60.6 \%$ correspondió a la pérdida económica como consecuencia del delito, el $35.8 \%$ al gasto en medidas preventivas y el 3.6\% restante al gasto en salud resultante de ser víctima de la delincuencia. De acuerdo con The Institute for Economics and Peace (2017), desde 2016, México es el segundo país menos pacífico de América Latina y desde 2007 ha perdido 48 lugares en el Índice Global de Paz.

\footnotetext{
5 Para México no existe una serie que sólo trate a los secuestros de los dueños o ejecutivos de empresas vinculadas a IED.

${ }^{6}$ Estrategia basada en operativos para capturar a los jefes de las principales organizaciones criminales (Benítez, 2009) con la idea de que al eliminarlos, las organizaciones colapsarían, pero en vez de ello las bandas se multiplicaron y han peleado crecientemente rutas y territorios, acentuando así la violencia.
} 
Ortiz et al. (2013) argumentan que mientras los homicidios dolosos afectan a la población en general, los secuestros tienen por objetivo primario - aunque no único - a agentes económicos específicos (dueños, empresarios y directivos) que tienen la capacidad de invertir y acumular capital. Así, aunque las condiciones de negocios sean favorables, el aumento de la probabilidad de ser víctima de la criminalidad podría tener más peso en la decisión del inversionista. ${ }^{7}$

Los dos delitos que aquí se analizan venían de niveles muy altos en $1997,{ }^{8}$ pero cayeron rápida y sostenidamente hasta 2005 . Justo a partir de la aplicación de la estrategia ya referida, ambas variables se dispararon notablemente. Véase figura 3.

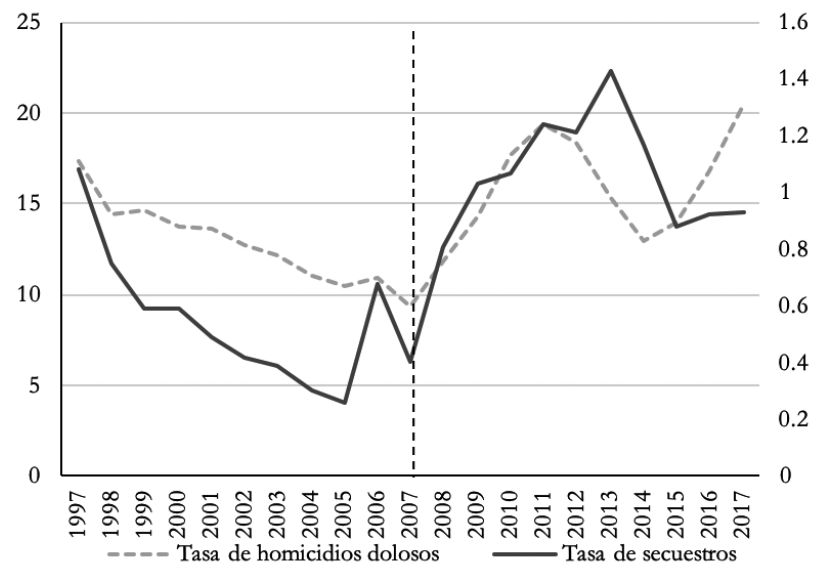

Figura 3. México: Tasas de secuestros y de homicidios, 1997-2017

\section{(Eventos por cada 100 mil habitantes)} Fuente: SESNSP (2017).

Nota: Tasa de secuestros en eje derecho y tasa de homicidios en el izquierdo.

En 2017, la tasa de homicidios (Hom) reportó la tasa históricamente más alta y la de secuestros (Sec) lo hizo en 2013. Sin embargo, debemos tomar en cuenta que ambas cifras están subestimadas. De acuerdo con la ENVIPE (2017), la "cifra negra" (delitos que no se denuncian) de Sec en 2012 fue del $98 \%$ y la de Hom del $30 \%$.

En términos de la hipótesis, observamos que en el mismo periodo, y según datos de la Secretaría de Economía (2017), a partir de 2007 las sociedades con capital extranjero que

\footnotetext{
${ }^{7}$ Porque no solo implica poner sus propias vidas en riesgo, sino también las de sus familias, y el costo de evitar o de sufrir este delito puede ser más alto que las ganancias de la inversión. De acuerdo con Consulta Mitofsky (2017), el secuestro es el delito que más temor genera en la población. Le siguen el robo a mano armada y los homicidios.

${ }^{8}$ Se inicia el análisis en 1997 porque es el primer año para el que existe información oficial disponible.
} 
registraron actividades comerciales o industriales en México han disminuido dramáticamente (figura 4) y, paralelamente, la IED nominal se ha estancado (figura 5) y su proporción a PIB ha caído desde 2001. La figura 5 muestra la evolución histórica de la IEDT nominal (en millones de dólares corrientes) desde 1970. Llama la atención que desde el año 2001 ha tenido mucha volatilidad y se ha estancado.

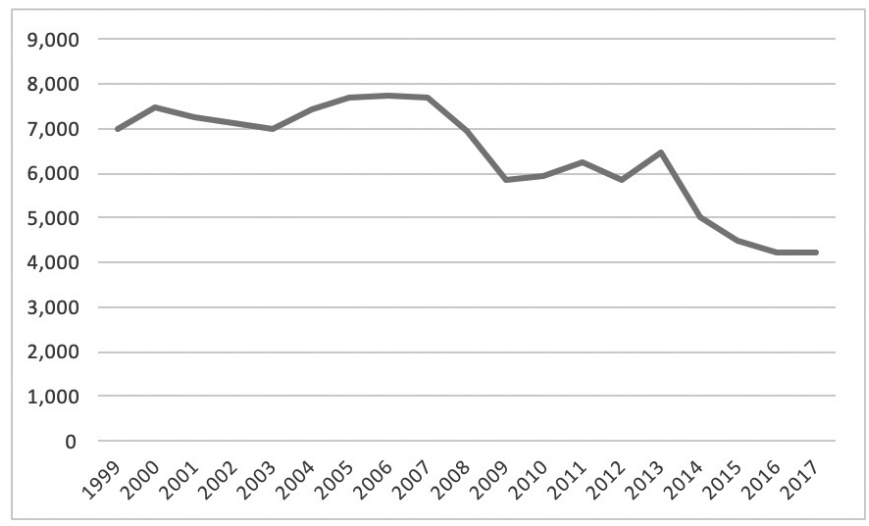

Figura 4. México: Número de sociedades con IED, 1999-2017

Fuente: Secretaría de Economía (2017).

Por otro lado, se observa que hay dos puntos de inflexión en su evolución histórica y que coinciden con: a) la promulgación del Reglamento de la Ley para Promover la Inversión Mexicana y Regular la Inversión Extranjera de 1989, b) la Ley de Inversión Extranjera de 1993 y el inicio del TLCAN en 1994.

Conviene hacer un análisis más preciso de lo ocurrido desde 2000. A partir de ese año ha habido tres importantes outlyers que corresponden a importantes compras de activos por empresas transnacionales: a) en 2001 la compra de Banamex por US \$12 mil 447 millones de dólares (mdd), y b) la compra de cerveceras en 2010 y 2012 por US $\$ 7,700$ mdd y US\$20.1 mil mdd, respectivamente. ${ }^{9}$

\footnotetext{
9 En consecuencia, es plausible considerar que estas inversiones difícilmente abonaron al crecimiento económico de esos años ni de los subsecuentes. Véanse CEFP (2002), Proceso (2010) y Lara y Espinosa (2012).
} 


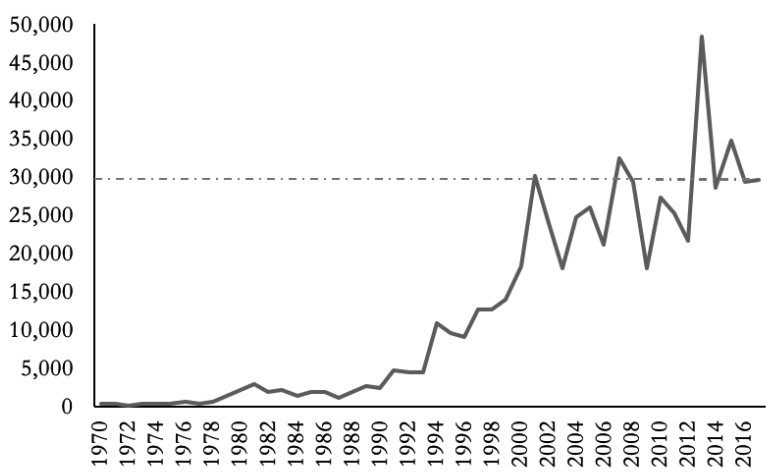

Figura 5.México: IEDT anual, 1970-2017(Millones de dólares corrientes)

Fuente: Secretaría de Economía (2017).

Por último, en la figura 6 se muestra la contribución relativa de los componentes de la IEDT. ${ }^{10}$ Llama la atención la abrupta caída de IEDNI que en 1997 representó el $71 \%$ y en 2017 solo el 38\%. La IEDR, por su parte, subió de $16.7 \%$ a $32.5 \%$, mientras que el componente de "cuentas entre compañías" creció notablemente al pasar de $12.2 \%$ a 29\% en el mismo periodo.

En suma, queda claro que el componente IEDNI, que representa al "capital fresco" y que es el que cumpliría con los efectos virtuosos de la inversión, ha perdido mucho peso, lo que apunta en favor de la hipótesis central del artículo.

Por otro lado, el aumento en el componente de "Nuevas Inversiones" podría explicarse porque aun con la violencia y la ausencia de estado de derecho, cerrar las plantas y salirse de México puede ser más costoso que absorber el costo de la criminalidad. Aun así, CEPAL (2018) advierte que desde 2011 ha caído la rentabilidad media de la IED en toda la región de América Latina, por lo que no se descarta que esta variable se resienta adicionalmente. ${ }^{11}$

\footnotetext{
${ }^{10}$ La IEDT se compone de 3 cuentas: a) Nuevas Inversiones (IEDNI), b) Reinversión de Utilidades (IEDR), y c) Cuentas Entre Compañías (Banco de México, 2017b). Se excluye del análisis a estas últimas ya que la conforman los préstamos de las matrices residentes en el exterior a sus filiales en México y las importaciones de activo fijo realizadas por empresas. De esta manera, consideramos que sólo las dos primeras reflejan más claramente el comportamiento de la IEDT de acuerdo con la hipótesis.

${ }^{11}$ Se añadió este comentario a pedido expreso de un árbitro. Si bien resultaría interesante medir más a fondo el impacto que la rentabilidad tiene y eventualmente tendría sobre la IED y sus componentes, no hay series con la misma periodicidad que la que usamos en este trabajo y rebasa por completo nuestro objetivo.
} 


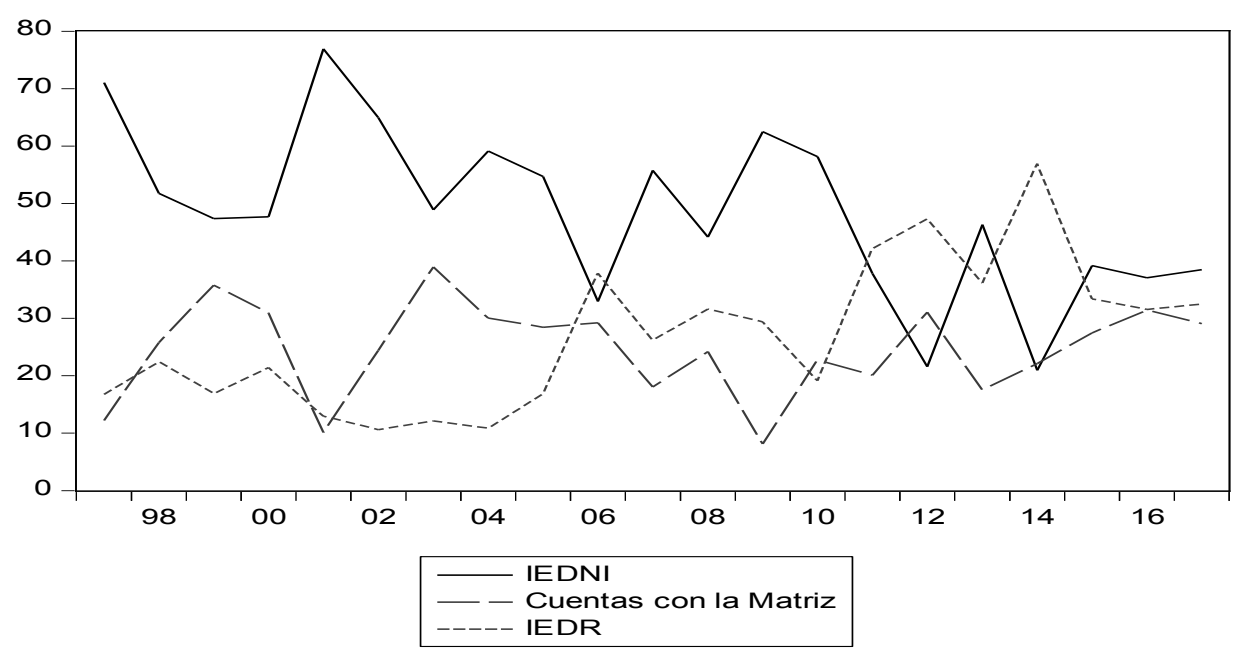

Figura 6. México: Componentes de la IEDT, \% del total, 1997-2017

Fuente: Cálculos propios basados en Banco de México (2017a).

\section{Aspectos econométricos}

Para probar la hipótesis central y, de acuerdo con la revisión de literatura, se eligió el siguiente conjunto de información (Y) para analizar los determinantes socioeconómicos de la IED en México (1997-2017):

$$
\mathrm{Y}=\{\text { IEDT, IEDNI, IEDR, Sec, Hom, PIB, CC }\}
$$

Donde: IEDT, IEDNI e IEDR son la Inversión Extranjera Directa Total y sus componentes de Nuevas Inversiones y Reinversiones, respectivamente (expresadas en miles de millones de US dólares, mmdd), Banco de México (2017a); Sec es la tasa de secuestros y Hom es la tasa de homicidios dolosos (ambos son eventos reportados por cada 100 mil habitantes), ENVIPE (2017); PIB es el Producto Interno Bruto de México (en miles de millones de pesos constantes de 2008), INEGI (2018); CC es la relación del saldo de la cuenta corriente a PIB (en pesos constantes) ${ }^{12}$ INEGI (2018) y Banco de México (2017a).

\footnotetext{
${ }^{12}$ Moosa (2002) define el riesgo país como la exposición a una pérdida económica en operaciones transnacionales. Dans (2012) y Dussel et al (2003) definen a CC como un indicador de riesgo país, lo que justifica su signo negativo a IEDT y sus componentes.
} 
La alta correlación existente entre Sec y Hom provocó que al incorporarlos como regresores simultáneos se obtuvieran signos incorrectos y falta de significancia estadística, ${ }^{13}$ por lo cual se estimaron dos modelos alternativos para cada variable de interés y a partir del Coeficiente de Theil y de la significancia estadística y económica se eliminaron dos, por lo que todo el análisis de inferencia se hizo a partir de la combinación de cuatro modelos robustos y correctamente especificados. Las series son no estacionarias en sus niveles, pero estacionarias en primeras diferencias, como se ve en la tabla $1 \mathrm{~A}$ del anexo. ${ }^{14}$

En la tabla 2A se prueba que existen relaciones de cointegración únicas (de equilibrio de largo plazo) para cada uno de los seis modelos estimados. Por la escasez de grados de libertad, todas las especificaciones tienen un rezago, por lo que en todos los casos se tienen VECM(1).

El enfoque moderno de series de tiempo sugiere que es necesario tener en cuenta no sólo los parámetros de largo plazo, sino también el desequilibrio de corto plazo dejando hablar libremente a los datos (Juselius, 2006 y Lütkepohl y Krätzig, 2004). Esta es una característica específica y muy importante de la metodología VEC.

De acuerdo con Patterson (2000), la condición necesaria para que estos modelos tengan solución es que al menos exista un vector de cointegración $(r=1)$ y que el sistema sea de rango reducido $(r<k)$. En la medida en que esto se cumpla, debe existir un mecanismo que asegure los ajustes dinámicos de corto plazo en términos de que los choques que sufre el sistema deben corregir la evolución de las variables hacia su relación de equilibrio de largo plazo en torno a la variable que hemos definido como dependiente.

El VEC general irrestricto de Johansen (1988) en forma reducida y sin considerar variables exógenas se puede expresar como:

$$
\Delta y_{t}=\alpha \beta^{\prime} y_{t-1}+\sum_{i=1}^{t-1} \Gamma_{i} \Delta y_{t-i}+\varepsilon_{t}
$$

Donde es un vector de variables endógenas (que se ha definido previamente como el conjunto de información, Y), es el mecanismo de corrección de error y sobre el que se prueba exogeneidad débil, es el número de relaciones (vectores) de cointegración, es la matriz de coeficientes del VAR de corto plazo en primeras diferencias, y es un vector de innovaciones de comportamiento Gaussiano.

Todos los modelos se estimaron en forma doble logarítmica, ${ }^{15}$ por lo que los parámetros estimados representan elasticidades constantes de largo plazo. ${ }^{16}$

Como se estimaron dos modelos para cada variable de interés, es importarte definir los criterios de selección. La econometría aplicada enfrenta siempre el problema sobre la selección del modelo "verdadero" o el que más se aproxima al Proceso Generador de Información,

\footnotetext{
${ }^{13}$ Loría y Salas (2016) demuestran la importancia y las consecuencias de la multicolinealidad en el caso de modelos VEC.

${ }^{14}$ Por la falta de grados de libertad no fue posible hacer pruebas de raíz unitaria con cambio estructural.

${ }^{15}$ Salvo para CC porque en general exhibe valores negativos.

${ }^{16} \mathrm{Y}$ semi elasticidades para el caso de CC.
} 
que es totalmente desconocido por el investigador (Hendry, 1995). En ese sentido, se debe buscar el mejor modelo estadístico estimable que, a fin de cuentas, deber ser aquel que sea más parsimonioso, plausible e informativo (Feldstein, 1982: 829). Esto se consiguió al seguir el procedimiento iterativo de "lo general a lo particular" de Hendry (1995). ${ }^{17}$

En este trabajo nos enfrentamos al problema de dos "modelos alternativos o rivales" por variable, por lo que para discernir y seleccionar el modelo que mejor prueba la hipótesis central, se siguió la siguiente estrategia: a) cumplir con los criterios habituales de correcta especificación, b) obtener los signos de los regresores que dicta la teoría (Hendry y Richard, 1983), c) que la simulación dentro de la muestra arrojara el menor componente sistemático (sesgo) del Coeficiente de Theil ${ }^{18}$ y d) que se cumpliera la "prueba en medio de los ojos" de Kennedy (2002). ${ }^{19}$

La normalización de los seis modelos sobre IED, IEDNI é IEDR es adecuada a partir de probar la exogeneidad débil (cuadro 3A), que es una exigencia de correcta especificación (Maddala, 1996, Charemza y Deadman, 1999 y Johansen, 1992). De no cumplirse esta condición, resultará que esa variable a su vez debe estimarse. Se encontró que esta condición no se cumple para CC en los modelos 1,5 y 6 , pero estimarla queda totalmente fuera del interés del artículo. ${ }^{20}$ Los modelos 2 y 4 sugieren que el PIB podría explicarse por las demás variables, lo que se comprueba con los impulsos-respuesta de los modelos 1 y 6 , que se presentan más adelante y abona en favor de la hipótesis central del artículo. Por último, Sec y Hom no son débilmente exógenos en los modelos 6 y 4, respectivamente, lo cual sugiere que habría que modelarlos específicamente, pero también rebasa el objetivo del artículo.

Finalmente, y para efectos de pronóstico, se requiere que además de cumplir con la condición de exogeneidad débil, haya causalidad en el sentido de Granger. De esta suerte, se cumplirá con la condición de exogeneidad fuerte (Charemza y Deadman, 1999). De acuerdo con la tabla 4A esta condición se cumple para los modelos 2,5 y 6 , pero por los resultados del modelo 2 (que se precisan más adelante) sólo es posible considerarla para los dos últimos.

En la tabla 1 se presentan los resultados de la estimación de los seis modelos.

\footnotetext{
${ }^{17}$ En específico, de aplicar la estrategia TTT (test, test, test).

${ }^{18}$ Pindyck y Rubinfeld (1991: 336-341).

19 “LLos signos de los coeficientes son los esperados? ¿Las variables son estadísticamente significativas? ¿Las magnitudes de los coeficientes son razonables? ¿Los resultados son consistentes con la teoría?”

${ }^{20}$ Además de que carece de sentido teórico en el análisis. Este resultado más bien parece ser de tipo estrictamente estadístico.
} 
Tabla 1

Modelos de Cointegración. Resultados

\begin{tabular}{|c|c|c|c|c|c|c|}
\hline & \multicolumn{2}{|c|}{ IEDT } & \multicolumn{2}{|c|}{ IEDNI } & \multicolumn{2}{|c|}{ IEDR } \\
\hline & Modelo 1 & Modelo 2 & Modelo 3 & Modelo 4 & Modelo 5 & Modelo 6 \\
\hline Constante & -24.03 & -22.00 & 1.01 & 0.12 & -51.22 & -49.80 \\
\hline Sec & -0.28 & & -0.38 & & & -0.10 \\
\hline Hom & & -0.01 & & -1.09 & -0.87 & \\
\hline $\mathrm{CC}$ & -0.07 & 0.01 & & & -0.36 & -0.23 \\
\hline PIB & 1.87 & 1.75 & 0.12 & 0.39 & 3.78 & 3.54 \\
\hline $\begin{array}{l}\text { Mecanismo de } \\
\text { corrección de } \\
\text { error } \alpha_{1} *\end{array}$ & $\begin{array}{c}-0.96 \\
(-2.3)\end{array}$ & $\begin{array}{c}-1.39 \\
(-4.97)\end{array}$ & $\begin{array}{c}-0.72 \\
(-1.88)\end{array}$ & $\begin{array}{c}-0.69 \\
(-1.84)\end{array}$ & $\begin{array}{c}-0.55 \\
(-3.21)\end{array}$ & $\begin{array}{c}-0.45 \\
(-1.79)\end{array}$ \\
\hline $\begin{array}{l}\text { Identificación } \\
\text { global } X^{2}, 95 \% \\
\text { confianza }\end{array}$ & $0.05(0.99)$ & $4.78(0.44)$ & $6.98(0.13)$ & $4.67(0.09)$ & $0.01(0.99)$ & $0.07(0.99)$ \\
\hline $\begin{array}{l}\text { Raíz máxima } \\
\text { del polinomio } \\
\text { característico }\end{array}$ & 0.51 & 0.76 & 0.76 & 0.76 & 0.76 & 0.83 \\
\hline Lütkepohl & $6.64(0.57)$ & $8.34(0.40)$ & $6.01(0.42)$ & $1.27(0.97)$ & $5.17(0.73)$ & $8.70(0.36)$ \\
\hline $\operatorname{LM}(6)$ & $23.19(0.10)$ & $14.65(0.54)$ & $11.34(0.25)$ & $6.75(0.66)$ & $14.24(0.58)$ & $17.30(0.36)$ \\
\hline White NC & $119.51(0.49)$ & $151.77(0.23)$ & $55.14(0.22)$ & $78.53(0.13)$ & $116.24(0.58)$ & $121.49(0.44)$ \\
\hline Coef. de Theil & 0.000 & 0.005 & 0.031 & 0.026 & 0.000 & 0.002 \\
\hline
\end{tabular}

Nota: Todos los modelos fueron identificados al aplicar restricciones conjuntas y vinculantes a los parámetros de corrección de error y de largo plazo; es decir, que cada variable individual y conjuntamente fueran significativas en cada modelo (Boswijk, 1995), lo que se prueba de manera conjunta con la prueba .

* Con $n=20$, el valor crítico al 95\% de confianza de la prueba t con una cola es 1.72 , por lo que en todos los casos se rechaza la hipótesis nula: (Greene, 1998: 865).

Los resultados anteriores, específicamente la prueba de "identificación global" representados conjuntamente por el estadístico, indican que la identificación de cada vector de cointegración de cada ecuación en términos de la selección de la variable dependiente es correcta; es decir, que la normalización sobre la variable dependiente, sobre la que se plantea la cointegración de cada ecuación es correcta. Asimismo, que hay significancia individual, medida por el estadístico t, de cada regresor por lo menos al 95\%. Esto es a lo que Boswijk (1995) denomina que las restricciones individuales y conjuntas son vinculantes. Al conseguirlo, se puede concluir que cada VEC está correctamente identificado. 


\section{Análisis y discusión de resultados}

Si bien todos los modelos cumplen con los supuestos de correcta especificación, por los criterios de selección ya mencionados se eliminó del análisis a los modelos 2 y 3 . El primero debido a que el coeficiente de corrección de error es explosivo y a que presenta signo positivo de CC, lo cual contradice el supuesto de estabilidad dinámica del modelo y la hipótesis de riesgo país, respectivamente. El modelo 3 se desechó debido a que reportó el coeficiente de Theil más alto a pesar de no presentar ningún otro problema estadístico o de análisis económico.

Por otro lado, se advierte que CC se excluyó en los modelos 3 y 4 debido a que se obtuvieron resultados contrarios a los hechos estilizados y a la teoría.

De esta manera, el análisis que sigue se hizo a partir de la combinación de los modelos restantes que en ningún momento presentaron resultados antagónicos. Más aún, en todos los casos se trató de resultados complementarios.

Los modelos 5 y 6 reportan una elasticidad muy alta de IEDR al PIB (3.78 y 3.54, respectivamente), lo que refleja varios hechos importantes: a) la dinámica del PIB - aunque modesta - ha contrarrestado el efecto negativo de la criminalidad sobre los tres componentes de IED, y b) muestra que IEDR es muy sensible a la evolución del mercado interno.

Los modelos 1 y 6, particularmente el primero, muestran el efecto negativo de Sec a IEDT e IEDR. En este sentido, a pesar de que es plausible pensar que Sec debería tener mayor impacto en todos los costos empresariales y personales, las elasticidades de IEDNI e IEDR respecto a Hom (modelos 4 y 5 ) resultaron ser mucho mayores.

Para analizar los efectos dinámicos se estimaron las funciones de impulso-respuesta. ${ }^{21}$ Todas las respuestas tienen los signos esperados, son significativas y permanentes, con la excepción de la respuesta del PIB a IEDR que si bien es positiva y significativa (figura 9), sólo perdura nueve periodos. En particular, los cuadrantes 1,1 de las figuras 7-9 prueban la hipótesis principal del trabajo: el efecto significativo negativo y permanente de Sec y Hom sobre los tres tipos de inversión extranjera. También se observa que esas variables tienen un claro efecto negativo y permanente sobre el PIB.

El carácter permanente del efecto de la violencia en la IED y el PIB puede explicarse porque no es un choque que los agentes asuman transitorio. Por el contrario, la violencia y la delincuencia son fenómenos sociales muy complejos cuya erradicación es complicada y

\footnotetext{
${ }^{21}$ En todos los casos las bandas de confianza se calcularon con el método de Bootstrap (método percentil) con mil iteraciones que permite obtener la desviación estándar de la muestra al 95\% de confianza. Los modelos VEC presentados están exactamente identificados tanto por las restricciones que son globalmente significativas (cuadro 1), como por las pruebas de cointegración (Johansen, 1995) que demuestran la presencia de un solo vector de cointegración (cuadro 2A) que, mediante la exogeneidad definida por la causalidad de Granger (cuadros 3A y 4A), permite la normalización de sobre la IED (Lütkepohl et al, 2004: 98). Por las condiciones anteriores, se utilizó el método de impulsos generalizados (que no se acumulan) para evitar la sensibilidad del sistema al ordenamiento de las variables (Pesaran y Shin, 1998).
} 
costosa, por lo que se puede asumir que se mantiene presente en una sociedad durante mucho tiempo influenciando las decisiones de inversión de largo plazo.

También llama la atención - y es lo más preocupante - la capacidad del crimen de auto reproducirse. En todos los casos, Sec y Hom muestran histéresis probablemente debido a la alta rentabilidad de las actividades ilícitas que disuade a quienes las llevan a cabo de dedicarse a otro tipo de actividades no ilegales, lo que también explica la permanencia del efecto de estas variables en la IED y en el PIB. Por otra parte, la respuesta dinámica de IEDR a Sec parece ser la mayor del sistema a pesar de que se estimó una elasticidad baja (-0.10, modelo 6). Por último, se observa que el PIB es muy importante para explicar la evolución dinámica de IEDR, pero no a la inversa. Esto puede deberse a que este tipo de inversión, en general, no aumenta el acervo de capital ya que en su mayor parte no amplía sustancialmente la capacidad productiva debido a que se canaliza a comprar activos y plantas ya existentes.

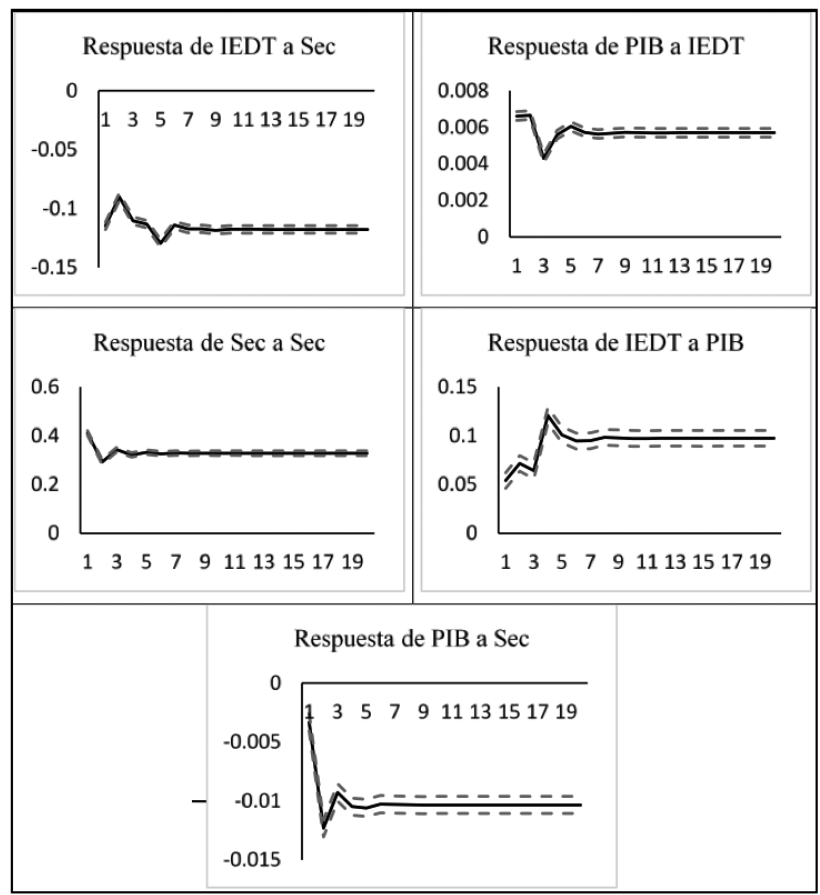

Figura 7. IEDT. Modelo 1. Análisis impulso-respuesta. Impulsos generalizados*

\footnotetext{
* A pedido de un árbitro, es pertinente señalar que en el marco de un modelo VEC no todas las respuestas son convergentes. En este caso, como se trata de procesos cointegrados no estacionarios, no existe representación de Wold porque se violan las condiciones de estabilidad dinámica de los modelos VAR, pero sí es posible calcular de la misma manera las matrices del análisis impulso respuesta $\left(\Phi_{\mathrm{s}}\right)$, Lütkepohl et al, (2004: 167).

Esta condición ocasiona que las respuestas $\left(\Phi_{\mathrm{s}}\right)$ no converjan a 0 , por lo que las respuestas pueden ser transitorias o permanentes (Lütkepohl et al, 2004: 168).
} 
E. Loría / Contaduría y Administración 65(3) 2020, 1-26

http://dx.doi.org/10.22201/fca.24488410e.2020.2246

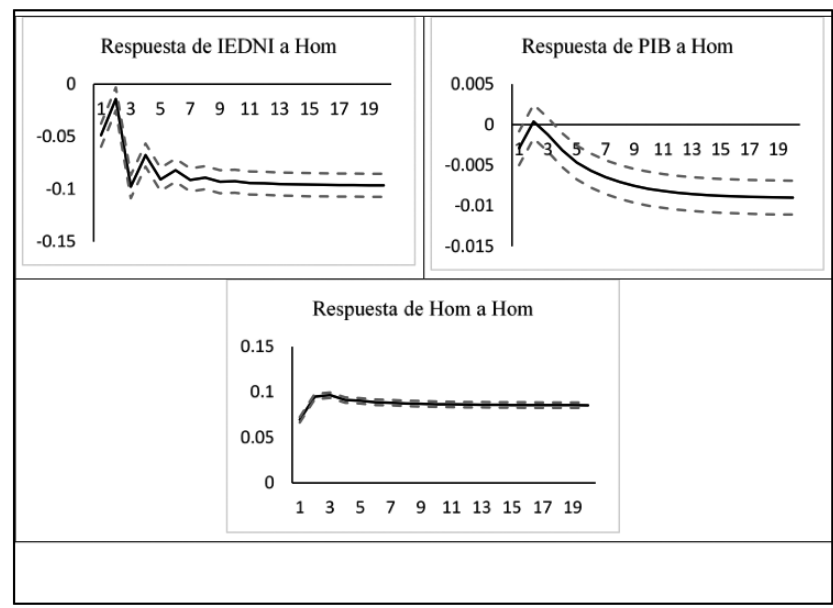

Figura 8.IEDNI. Modelo 4. Análisis impuso-respuesta. Impulsos generalizados

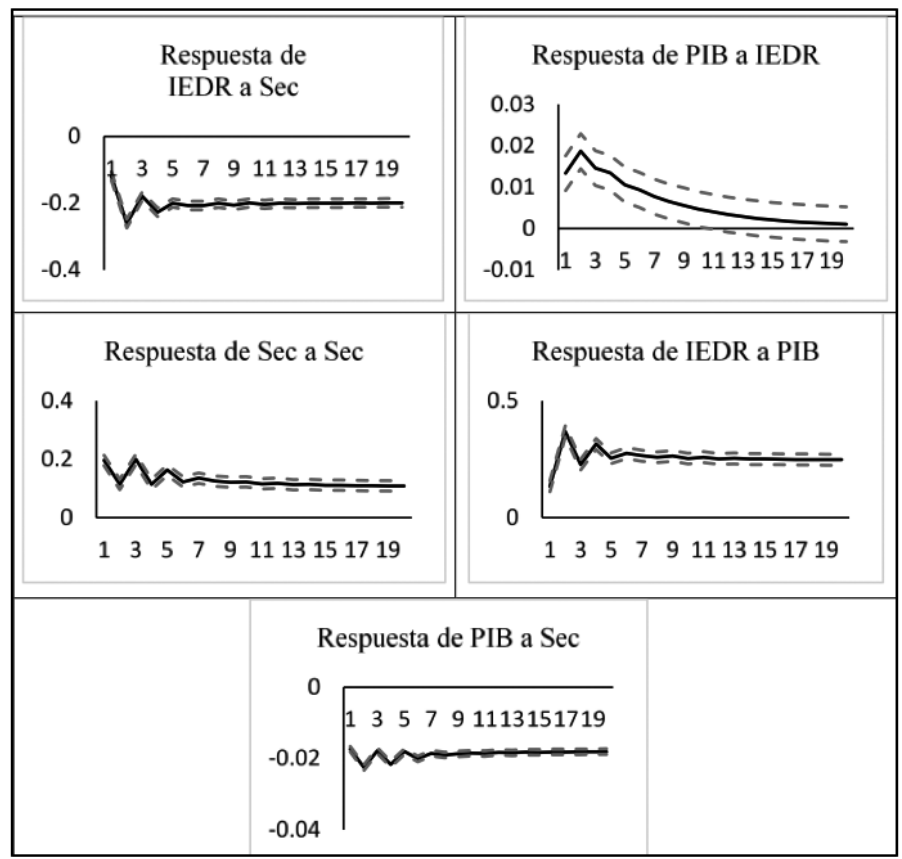

Figura 9. IEDR. Modelo 6. Análisis impulso-respuesta.Impulsos generalizados 


\section{Conclusiones}

La teoría económica, independientemente de su fundamentación o afiliación epistemológica, acepta que la inversión es la variable clave del crecimiento económico.

Si bien la IEDT no representa una proporción importante del PIB en México (alrededor del $3 \%$ desde el año 2000), sus flujos hacia el país pueden darnos una idea de qué tan favorable ha sido el ambiente para invertir.

Se observa que desde principios de los años 90 hubo un incremento importante en esta variable que se explica básicamente por la entrada en vigor del TLCAN. Después del año 2000 se ha estancado en términos nominales y ha caído como proporción del PIB a pesar de la ola de reformas estructurales que se han aplicado, de la estabilidad macroeconómica y de la gran integración económica con Norteamérica.

Los resultados de esta investigación son consistentes con la literatura reciente consultada en el sentido de que la estabilidad social, la institucionalidad y la seguridad cada vez inciden de manera más importante en la entrada de IED a un país.

La violencia que al menos desde hace una década azota a México impacta la paz social, pero también acarrea problemas económicos y la IED se ha visto afectada a partir del incremento de los delitos de alto impacto medidos a través de las tasas de secuestros y de homicidios dolosos.

Esta violencia manda señales claras que desincentivan a los inversionistas y a los mercados internacionales y provoca que los macro fundamentales pasen a segundo plano.

A través del método de cointegración de Johansen (1988) se demuestra empíricamente el efecto negativo y permanente que tienen los secuestros y los homicidios dolosos sobre la IEDT y sus dos componentes principales (nuevas inversiones, IEDNI y reinversiones, IEDR) y sobre el PIB. La permanencia de este efecto se explica por la histéresis que presentan las tasas de secuestros y homicidios y esta, a su vez, se explica por la alta rentabilidad que reportan este tipo de actividades.

Se encontró que los homicidios afectan a IEDNI con una elasticidad de -1.09 y los secuestros a IEDT con una elasticidad -0.28 .

Todos los resultados econométricos son concluyentes y congruentes con la hipótesis central, con lo que una conclusión crucial que se deriva del trabajo es que reestablecer el estado de derecho es prioritario para elevar la inversión extranjera directa total y su componente de nuevas inversiones y así reactivar el crecimiento económico del país. 


\section{Agradecimientos}

Centro de Modelística y Pronósticos Económicos, Cubículo 305, Facultad de Economía, UNAM. Circuito Interior s/n, Ciudad Universitaria, Edificio B, CDMX, C.P. 04510. Correo electrónico: eduardol@unam.mx. Agradezco los valiosos comentarios de Alfredo Coutiño y de Víctor Torres y la gran asistencia técnica de Raúl Cossío, Javier Valdez y Emmanuel Salas. Asimismo, agradezco los comentarios de los árbitros de la revista. Cualquier error que prevalezca es de mi entera responsabilidad. Este artículo es parte del proyecto de investigación Política monetaria y precarización del mercado laboral en México, una explicación alternativa al lento crecimiento 2000-2020 (IN300218), DGAPA, UNAM.

\section{Referencias}

Abbas, S. \& El Mosallamy, D. (2016). Determinants of FDI Flows to Developing Countries: An Empirical Study on the MENA Region. Journal of Finance and Economics, 4(1), 30-38.

Agyapong, D., Asiamah, M. \& Addo-Danquah, M. (2016). Organised Crime, Foreing Direct Investment and Economic Growth in Ghana. British Journal of Economics, Management \& Trade, 15(4), 1-12. DOI: https://doi. org/10.9734/bjemt/2016/29495

Aitken, B., Hanson, G. H. \& Harrison, A. E. (1994). Spillovers, Foreing Direct Investment and Export Behavior. NBER Working Paper Series No. 4967, 1-40. DOI: https://doi.org/10.3386/w4967

Alaimo, V., Fajnzylber, P., Guasch, L., López, J. H. \& Oviedo, A. M. (2009). The Investment Climate in Latin America, in Fajnzylber, L. Guasch, \& J. López (Edits.), Does the Investment Climate Matter? Microeconomic Foundations of Growth in Latin America (pp. 49-112). Washington: The World Bank. DOI: https://doi. org/10.1596/978-0-8213-7411-5

Banco de México (2017a). Inversión Extranjera Directa. Disponible en http://www.banxico.org.mx/SieInternet/ consultarDirectorioInternetAction. do?accion=consultarCuadro\&idCuadro=CE131\&sector=1\&locale $=$ es

Banco de México (2017b). Publicaciones y Prensa. Obtenido de Implementación de la Sexta Edición del Manual de Balanza de Pagos del FMI: Disponible en http://www.banxico.org.mx/apps/sie/\%7BC3EAAA53-9C11-465B6214-5FB87601C6ED\%7D.pdf

Becker, G. (1968). Crime and Punishment: An Economic Approach. Journal of Political Economy, 76(2), $169-217$. DOI: https://doi.org/10.1086/259394

Benítez, R. (2009). México 2010: Crimen organizado, seguridad nacional y geopolítica, en R. Benítez, A. Rodríguez, y A. Rodríguez (Edits.). Atlas de la seguridad y la defensa de México 2009. México: Colectivo de Análisis de la Seguridad con Democracia, 9-30.

Bernal, M. y Castillo, R. (2012). Efecto de la delincuencia sobre la inversión extranjera directa en México. Comercio Exterior, 62(3), 18-27.

Blomberg, S. Brock \& Mody, A. (2005). How Severely Does Violence Determine International Investment? Disponible en https://www.econstor.eu/handle/10419/31451

Boswijk, H. P. (1995). Efficient inference on cointegration parameters in structural error correction models. Journal of Econometrics, 69(1), 133-158. DOI: https://doi.org/10.1016/0304-4076(94)01665-m 
Busse, M. (2005). Measuring Political Risk: Risks to Foreign Investment. Transnational Corporations, 14(3), 149154. DOI: https://doi.org/10.4324/9781315249414

CEFP (2002). Comportamiento de la Inversión Extranjera Directa en México: 2001-2002/II. Disponible en http:// www.cefp.gob.mx/intr/edocumentos/pdf/cefp/cefp0262002.pdf

CEPAL (2018). La Inversión Extranjera Directa en América Latina y el Caribe. Organización de las Naciones Unidas (ONU). Disponible en https://repositorio.cepal.org/bitstream/handle/11362/43689/13/S1800684_es.pdf

Cevis, I. \& Camurdan, B. (2009). The Economical Determinants of Foreign Direct Investment (FDI) in Developing Countries and Transition Economies. e-Journal of New World Sciences Academy, 4(3), 210-213.

Charemza, W. \& Deadman, D. (1999). New Directions in Econometrics Practice. General to Specific Modeling, Cointegration and Vector Autoregression, $2^{\text {nd }}$ ed. United Kingdom: Edward Elgar.

Consulta Mitofsky (2017). XVII Encuesta Nacional sobre Percepción de Inseguridad en México. Disponible en http://www.consulta.mx

Dans, N. (2012). El riesgo país en la inversión extranjera directa: concepto y modalidades de riesgo. Papeles de Europa, año 2012, 25, 109-129. DOI: https://doi.org/10.5209/rev_pade.2012.n25.41100

Dellis, K., Sondermann, D. \& Vansteenkiste, I. (2017). Determinants of FDI Inflows in Advanced Economies: Does the Quality of Economic Matter? ECB Working Paper 2066, 1-26.

Dussel, E., Galindo, L. y Loría, E. (2003). Condiciones y efectos de la inversión extranjera directa y el proceso de integración regional en México durante los noventa. México: Plaza y Valdés, S.A. de C.V., UNAM, BID-Intal.

Enders, W. (2004). Applied Econometric Time Series. Estados Unidos: JohnWiley and Sons Inc.

ENVIPE. (2017). Encuesta Nacional de Victimización y Percepción sobre Seguridad Pública. México: INEGI. Disponible en http://www.beta.inegi.org.mx/contenidos/proyectos/enchogares/regulares/envipe/2017/doc/envipe2017_presentacion_nacional.pdf Revisado 26 de julio de 2018.

Esquivel, G. y Larraín, F. (2001). ¿Cómo atraer la inversión extranjera directa? Proyecto Andino de la Universidad de Harvard y la Corporación Andina de Fomento.

Fajardo, M. y de Jesús, L. (2015). Inversión extranjera directa en América Latina: una revisión en los albores del siglo XXI. Centro Regional de Investigaciones Multidisciplinarias. DOI: https://doi.org/10.22201/ crim.9786070291272e.2017

Feldstein, M. (1982). Inflation, Tax Rules and Investment: Some Econometric Evidence. Econometrica, 50(4), 393423. DOI: https://doi.org/10.3386/w0577

Findlay, R. (1978). Relative Backwardness, Direct Foreign Investment and Transfer of Technology: A Simple Dinamic Model. The Quality Journal of Economics, 92(1), 1-16. DOI: https://doi.org/10.2307/1885996

Greene, W. H. (1998). Análisis Econométrico. $3^{\mathrm{a}}$ ed. España: Prentice Hall.

Guerra, A. (2001). Factores determinantes de la inversión extranjera: introducción a una teoría inexistente. Comercio Exterior, septiembre, 825-832.

Hendry, D. \& Richard, J. (1983). The Econometric Analysis of Economic Time Series. International Statistical Review, 51(2), 111-163. DOI: https://doi.org/10.1093/0198293542.003.0018

Hendry, D. F. (1995). Dynamic Econometrics. Oxford University Press. DOI: https://doi.org/10.1093/019828316 4.001 .0001

Hernández, J. C. (2014). Análisis de la violencia en México. Instituto Tecnológico Autónomo de México, Tesis de licenciatura, México.

INEGI (2018). Banco de Información Económica. Disponible en http://www.inegi.org.mx/ sistemas/bie/

Institute for Economics and Peace. (2017). Mexico Peace Index. New York: IEP. Disponible en http://visionofhumanity.org/app/uploads/2017/04/Mexico-Peace-Index-2018-English-003.pdf

Johansen, S. (1988). Statistical Analysis of Cointegration Vectors. Journal of Economic Dynamics and Control, 12(2), 231-254. DOI: https://doi.org/10.1016/0165-1889(88)90041-3

Johansen, S. (1992). Testing weak exogeneity and the order of cointegration in UK money demand data. Journal of Policy Modeling,14(3), 313-334. DOI: https://doi.org/10.1016/0161-8938(92)90003-u 
Johansen, S. (1995). Likelihood-based inference in cointegrated vector autoregressive models. United Kingdom: Oxford University Press.

Juselius, K. (2006). The Cointegrated VAR model. United Kingdom: Oxford University Press. DOI: https://doi. org/10.1093/acrefore/9780190625979.013.247

Kennedy, P. E. (2002). Sinning in the Basement: What are the Rules? The Ten Commandments of Applied Econometrics. Journal of Economic Surveys, 16(4), 569-589. DOI: https://doi.org/10.1111/1467-6419.00179

Kotrajaras, P. (2010). Foreign Direct Investment and Economic Growth: A Comparative Study among East Asian Countries. Applied Economics Journal, 17(2), 12-26.

Lara, P. y Espinosa, E. (2012). Grupo Modelo fue adquirida por AB InBev. Excelsior. Disponible en https://www. excelsior.com.mx/2012/06/29/dinero/844365\#imagen-2

Loría, E. \& Salas, E. (2016). A SVEC Model to Forecast and Perform Structural Analysis (Shocks) for the Mexican Economy, 1985Q1-2014Q4, I, in Rojas, I. \& H. Pomares. Time Series Analysis and Forecasting. Springer. DOI: https://doi.org/10.1007/978-3-319-28725-6_11

Lütkepohl, H. \& Krätzig, M. (2004). Applied Time Series Econometrics. United Kingdom: Cambridge University Press. DOI: https://doi.org/10.1017/cbo9780511606885

Maddala, G. (1996). Introducción a la Econometría. $2^{\mathrm{a}}$ edición. México: Prentice-Hall.

MacKinnon, J. G., Haug, A. \& Michelis, L. (1999). Numerical Distribution Functions of Likelihood Ratio Tests for Cointegration. Journal of Applied Econometrics, 14(5), 563-577. DOI: https://doi.org/10.1002/(sici)1099-125 5(199909/10)14:5<563::aid-jae530>3.3.co;2-i

Madrazo, F. (2009). The Effect Of Violent Crime On FDI: The Case Of Mexico 1998-2006. Washington: Georgetown University.

Molzahn, C., Ríos, V. \& Shirk, D. (2012). Drug Violence in Mexico. Data and Analysis Through 2011. San Diego: Trans-Border Institute. DOI: https://doi.org/10.1007/s12117-010-9096-7

Moosa, I. A. (2002). Foreign Direct Investment: Theory and Practice. United Kingdom: Springer.

Oladipo, S. (2013). Macroeconomic Determinant of Foreign Direct Investment in Nigeria (1985-2010): a GMM Approach. Journal of Emerging Issues in Economics, Finance and Banking, 2(4), 801-817.

Ortiz, C., Uribe, J. y Vivas, H. (2013). Productividad, acumulación y deseconomías públicas en el crecimiento económico colombiano. Cuadernos de Economía, 32(59), 235-266.

Patterson, K. D. (2000). An Introduction to Applied Econometrics. United States: St. Martin's Press.

Pesaran, H. \& Shin, Y. (1998). Generalized Impulse Response Analysis in Linear Multivariate Models. Economics Letters, 58(1), 17-29. DOI: https://doi.org/10.1016/s0165-1765(97)00214-0

Pindyck, R. \& Rubinfeld, D. (1991). Econometric Models \& Economic Forecasts. $3^{\text {rd }}$ ed. McGraw-Hill International Editions.

PNUD. (2013). Informe Regional de Desarrollo Humano 2013-2014. Nueva York: ONU. Disponible en http:// www.latinamerica.undp.org/content/dam/rblac/img/IDH/IDH-AL\%20Informe\%20completo.pdf

Proceso. (2010). FEMSA vende a Heineken su división cervezas y adquiere $20 \%$ de la firma holandesa. Proceso. Disponible en https://www.proceso.com.mx/108048/femsa-vende-a-heineken-su-division-cervezas-y-adquire-20-de-la-firma-holandesa

Pyshval, R. \& Suárez, G. (2006). 'Captive Markets': The Impact of Kidnappings on Corporate Investment in Colombia. Coyuntura Económica. XXXVI, 1. DOI: https://doi.org/10.2139/ssrn.910886

Ríos-Morales, R. y O’Donovan, D. (2006). ¿Pueden los países de América Latina y el Caribe emular el modelo irlandés para atraer inversión extranjera directa? Revista de la CEPAL, abril (88), 51-70. DOI: https://doi. org/10.18356/4fa64de1-es

Rizzo, M. (1979). The Cost of Crime to Victims: An Empirical Analysis. The Journal of Legal Studies, 8(1), 177205. 
Secretaría de Economía. (2017). Estadística oficial de Inversión Extranjera Directa. Disponible en http://www. economia.gob.mx/comunidad-negocios/competitividad-normatividad/inversion-extranjera-directa/estadistica-oficial-de-ied-en-mexico

SESNSP. (2017). Incidencia Delictiva. Dispobible en http://www.incidenciadelictiva. secretariadoejecutivo.gob. $\mathrm{mx} /$

Torres, V. y Polanco, M. (2015). Inversión extranjera directa y criminalidad en las regiones de México. Economía Actual, 8(2), 14-22.

UNCTAD. (2017). World Investment Report. United Nations Organization. Disponible en http://unctad.org/en/PublicationsLibrary/wir2017_en.pdf

UNDOC. (2007). Crime, Violence, and Development: Trend, Costs and Policy Options in the Caribbean. Report No. 37820. Disponible en https://www.unodc.org/pdf/research/Cr_and_Vio_Car_E.pdf

Vittorio, D. \& Marani, U. (2008). Organized Crime and Foreing Direct Investment: the Italian Case. Working Paper, Dipartimento DOPES. Universita Magna Graecia di Catanzaro. DOI: https://doi.org/10.1007/s12117008-9039-8

Waldkirch, A. (2010). The Effects of Foreign Direct Investment in Mexico since NAFTA. World Economy, 33(5), 710-745. DOI: https://doi.org/10.2139/ssrn.1115300

World Justice Project. (2018). Índice de Estado de Derecho 2017-2018. Washington D.C., USA. 


\section{Anexo}

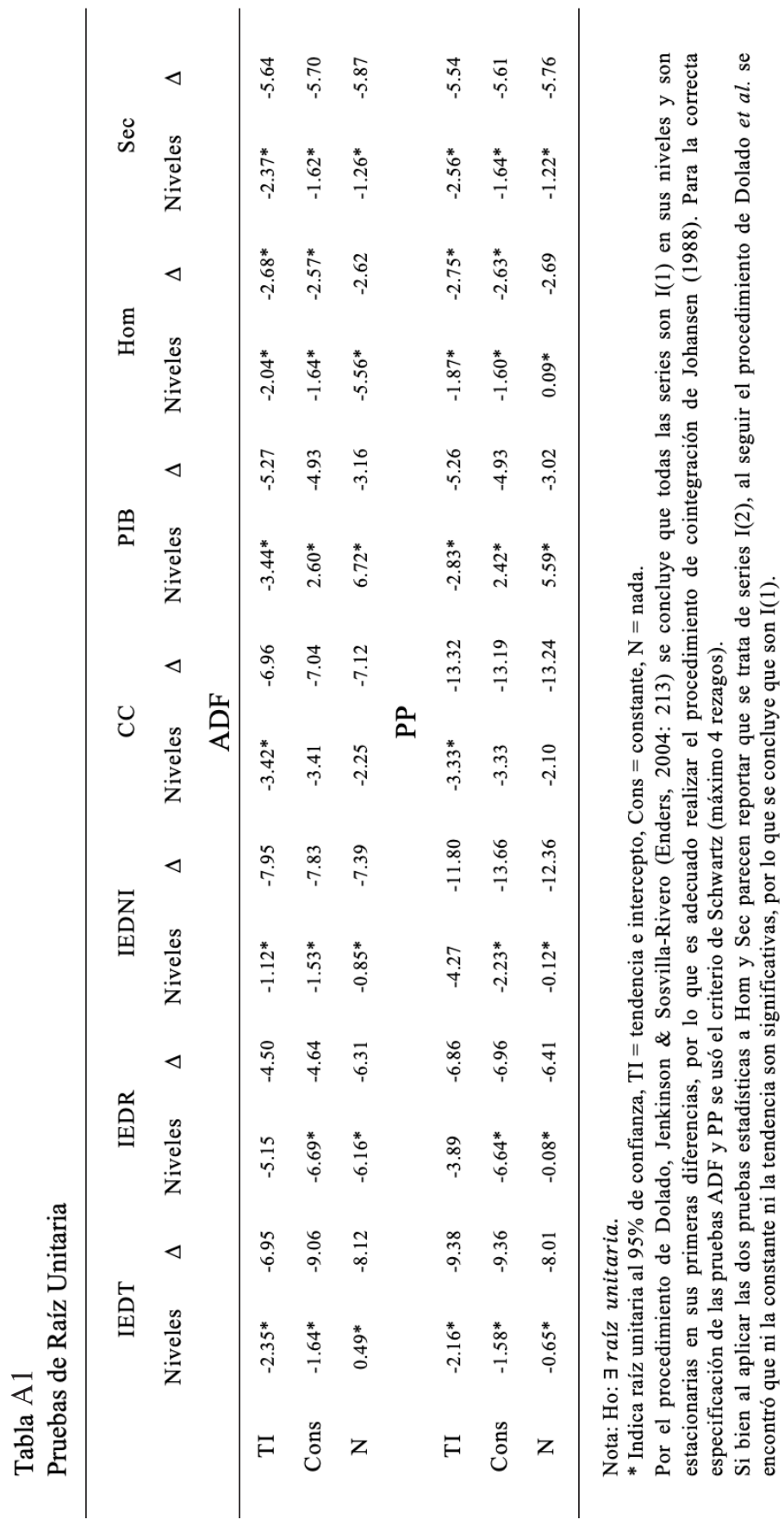




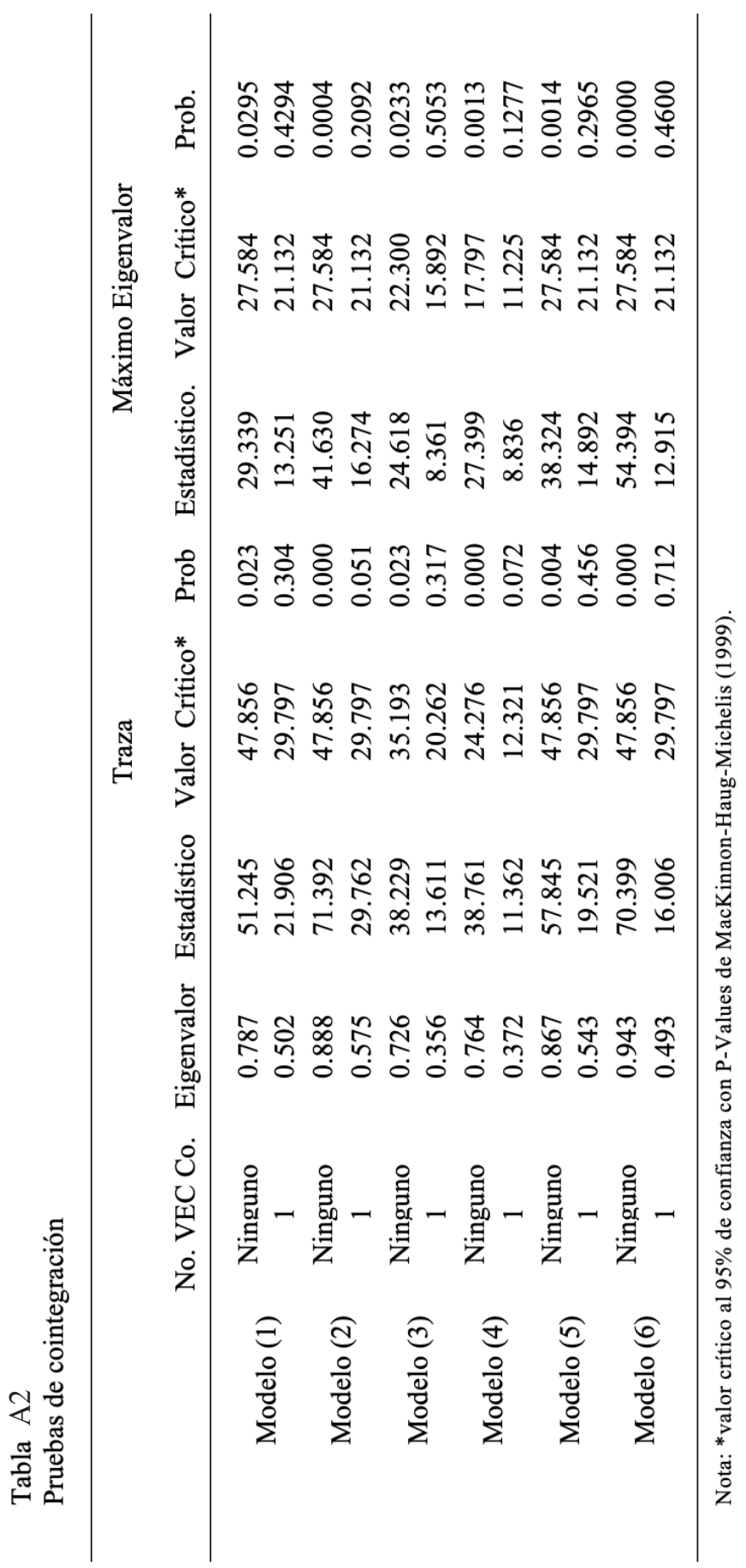


Tabla A3

Exogeneidad débil

\begin{tabular}{ccccccc}
\hline & \multicolumn{2}{c}{ IEDT } & \multicolumn{2}{c}{ IEDNI } & \multicolumn{2}{c}{ IEDR } \\
& Modelo 1 & Modelo 2 & Modelo 3 & Modelo 4 & Modelo 5 & Modelo 6 \\
$\alpha_{\text {Sec }}$ & $0.03(0.85)$ & & $0.04(0.83)$ & & & $15.94(0.00)$ \\
$\alpha_{\text {Hom }}$ & & $1.28(0.25)$ & & $5.20(0.02)$ & $0.30(0.58)$ & \\
$\alpha_{\text {CC }}$ & $6.55(0.01)$ & $3.02(0.08)$ & & & $23.13(0.00)$ & $24.07(0.00)$ \\
$\alpha_{\text {PIB }}$ & $0.20(0.65)$ & $5.67(0.01)$ & $2.31(0.12)$ & $9.62(0.00)$ & $1.16(0.28)$ & $0.32(0.56)$ \\
\hline
\end{tabular}

Nota: $H o: \alpha_{i}=0 *$ denota $H o$ se cumple al 95 de confianza.

Tabla A4

Pruebas de Causalidad en el Sentido de Granger

\begin{tabular}{ccccc}
\hline & Modelo & Chi-sq & df & Prob. \\
IEDT & 1 & 3.717305 & 3 & 0.2937 \\
& 2 & 16.89627 & 3 & 0.0007 \\
IEDTNI & 3 & 1.213098 & 2 & 0.5452 \\
& 4 & 1.019145 & 2 & 0.6008 \\
IEDR & 5 & 26.69855 & 3 & 0.0 \\
& 6 & 16.17133 & 3 & 0.00100 \\
\hline
\end{tabular}

Nota: $H_{o}$ : $\nexists$ causalidad en el sentido de Granger. Las variables están en primeras diferencias dentro del VAR de cointegración con lo cual -y por construcción- se evita el problema de espuriedad. 